Case Report

\title{
M ONOARTICULAR SYNOVITIS OF KNEE DUE TO UNRECOGNISED INTRA-ARTICULAR PLANT SPLINTER
}

\author{
Siddharth M. Shetty ${ }^{1}$, Vikram Shetty ${ }^{2}$, Arjun Ballal ${ }^{3}$ \& Jayaprakash Shetty ${ }^{4}$ \\ ${ }^{1,2}$ Associate Professors, ${ }^{3}$ Post Graduate, ${ }^{4}$ Professor, Department of Orthopaedic Surgery, \\ K.S. Hegde M edical Academy, Nitte University, Mangalore - 575 018, India. \\ Correspondence : \\ Arjun Ballal \\ Post Graduate, Department of Orthopaedic Surgery, K.S. Hegde Medical Academy, \\ Nitte University, Mangalore - 575 018, India. \\ Mobile : +91 8431644077 E-mail : arjchess_Ip@rediffmail.com
}

\begin{abstract}
:
Synovitis of a knee secondary to penetrating splinter injuries of plant origin aren't frequently reported. Historically, it is considered aseptic and treated with removal of the intraarticular foreign body and affected synovial lining.

We report a case of a 59-year-old healthy man whose left knee wasinjured while at work at a bamboo plantation site. He presented to us with pain and swelling of the knee for one month. He underwent diagnostic arthroscopy of the knee wherein a foreign body was identified, located and removed and near total synovectomy was done.
\end{abstract}

We present this patient's case and its management with a review of the relevant literature.

Keywords : Synovitis, plant splinter, knee swelling, arthroscopy, foreign body

\section{Introduction:}

Monoarticular synovitis of knee secondary to penetrating splinter injuries of plant origin are rare but a well described entity which is usually under-recognised ${ }^{1,2,3}$ This is apparently is due to the trivial nature of trauma and a very insidious onset of symptoms. The effusion may be sterile and is due to the irritation of the foreign body to the synovial tissue ${ }^{3,4}$ but occasionally organisms may be isolated. ${ }^{5}$

Radiographs may be negative and M RI may show synovitis without a specific pathology. ${ }^{6}$

Arthroscopy is the most important diagnostic and therapeutic procedure along with radiographic examination in a case of suspicious history and clinical features. ${ }^{7}$

\begin{tabular}{|c|}
\hline Access this article online \\
\hline Quick Response Code \\
\hline
\end{tabular}

\section{Case Report:}

A 59 year old gentleman presented to us with alleged history of penetrating trauma at a bamboo plantation site when accidentally his sickle cut a wound over the anterior aspect of the knee one month ago. The wound had healed within two weeks without any complications but the knee pain and swelling which gradually progressed with disturbing his daily activities and sleep. He complained of throbbing type of pain, which was not relieved with analgesics and rest. He had no history of fever or chills or any otherjoint involvement.

He was clinically assessed and effusion was noted the knee joint (Figure 1), with a fixed flexion deformity and terminally painful range of motion with local rise in temperature. The routine haematological evaluation was normal (total count: 9200, CRP: Negative) and no infectious pathology could be recognised.

X-ray was within normal limits with early features of osteoarthritis. MRI images showed significant effusion in the knee. Diagnostic arthroscopy of the left knee was performed under spinal anaesthesia. There was pale yellow colour clear synovial fluid about $120 \mathrm{ml}$ which was drained and sent for evaluation (Figure 2). Synovial tissue was inflamed with features of unhealthy fimbriae. A brown coloured 3-4 mm long slender foreign body was identified 
in the medial joint space overlying the meniscus (Figure 3) and was extracted (Figure 4). No other foreign bodies were recognised on probing the joint. Near total synovectomy of the knee was done.

\section{Closure of the surgical site was done.}

The synovial fluid analysis revealed, normal sugar and proteins. The culture reported no growth in the fluid sample. Intravenous antibiotics were stopped at five doses.

Knee exercises were started immediate post op and full range of motion was attained in two months and the operated knee appeared normal like the contralateral knee on review (Figure 5).

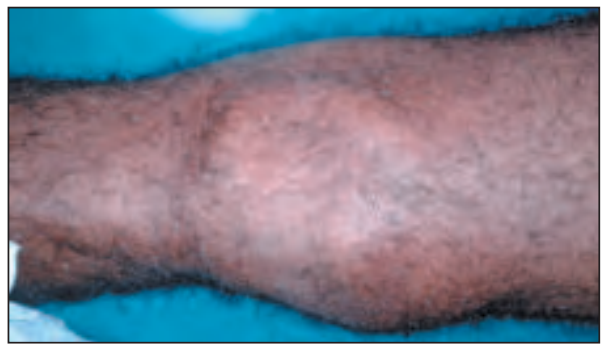

Figure 1: Left knee showing suprapatellar and parapatellar effusion.

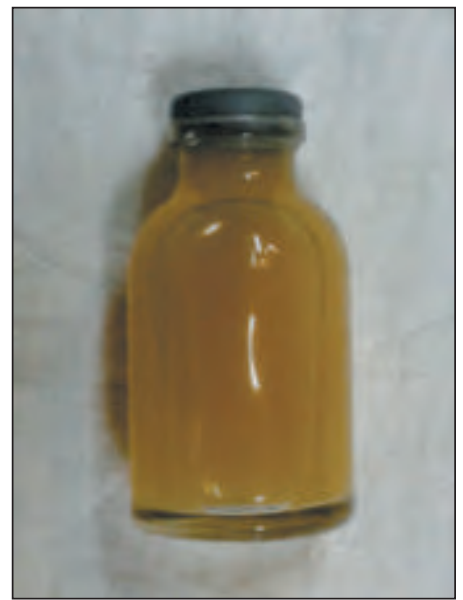

Figure 2 : Pale yellow synovial fluid drained from the knee.

\section{Discussion and Review of Litrature:}

Arthritis caused by plant thorn penetration is well known, especially among children. Blackthorn or date palm thorns caused synovitis is reported in most cases ${ }^{8}$, but it can occur from thorns of several kind of plants ${ }^{9,10,11}$. The most commonly affected joint is the knee, but similar processes

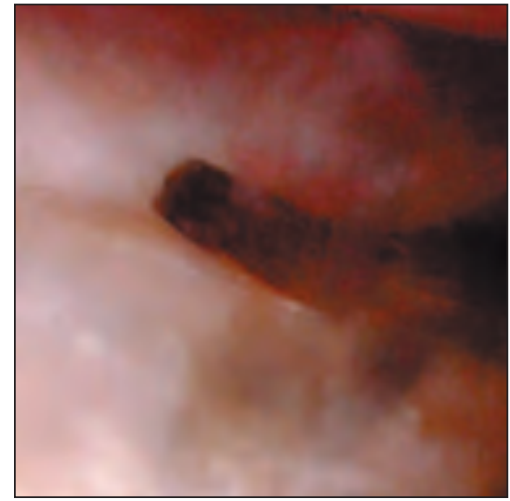

Figure 3: 3-4 mm long slender foreign body identified in the medial joint space overlying the meniscus.

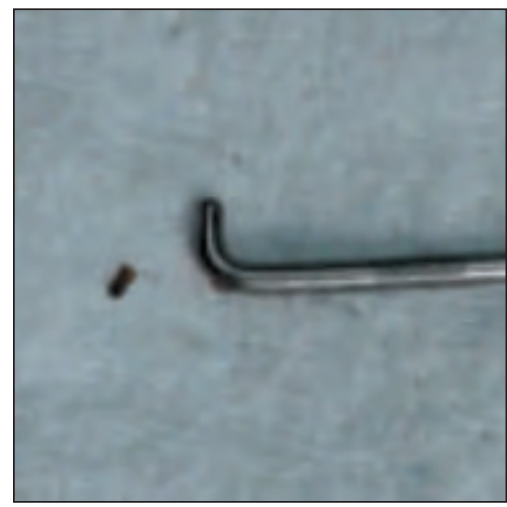

Figure 4 : $4 \mathrm{~mm}$ long slender foreign body after removal from the knee.

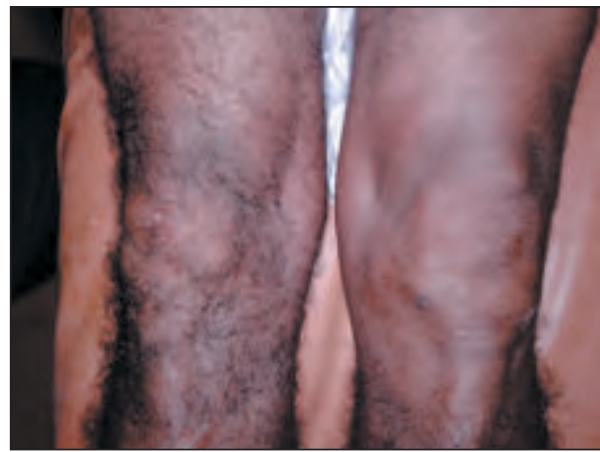

Figure 5 : At 6 weeks post-op the operated knee appears normal just like the contralateral knee

have been described in the hand, ankle, and wrist ${ }^{9,0,12,13}$.

Historically, failure to discover an organism in the joint fluid after a plant thorn injury led to the hypothesis, first published in 1953, that the synovitis after these injuries was an aseptic inflammatory reaction provoked by alkaloid compounds in the vegetable matter ${ }^{14}$. This disease at that 
time was known as "blackthorn inflammation" ${ }^{15}$.

It was not until 1977 that the first positive culture specimen of synovial fluid after plant thorn injury was reported with growth of Staphylococcus albus, Streptococcus hemolyticus, and Gram-negative rods ${ }^{16}$. Joint infection caused by Pantoeaagglomerans was first reported in $1978^{17}$

In earlier reports, treatment consisted of arthrotomy and removal of all thorn fragments ${ }^{14,15,16}$. In 1980, the advantages of arthroscopy became clear ${ }^{18}$. Arthroscopy may allow complete observation of the joint and extraction of the foreign body, but there still are several pitfalls. Plant fragments usually are too small to be seen and a reactive hypertrophic plica can obscure the presence of an intraarticular foreign body and may be missed by the

\section{References:}

1. Cahill N, King JD. Palm thorn synovitis. J Pediatr Orthop. 1984;4:175-179.

2. Cracchiolo A, Goldberg L. Local and systemic reactions to puncture injuries by the sea urchin spine and the date palm thorn. Arthritis Rheum. 1977;20:1206-1212.

3. Karshner RG, Hanafee W. Palm thorns as a cause of joint effusion in 17 children. Radiology. 1953;60:592-595.

4. Kelly JJ. Blackthorn inflammation. J Bone Joint Surg Br. 1966;48:474-477.

5. Sugarman M, Stobie DG, Quismorio FP, Terry R, Hanson V. Plant thorn synovitis. Arthritis Rheum. 1977;20:1125-1128.

6. Said HG, Masoud MA, Yousef HA, Imam HM. Multidetector CT for thorn (wooden) foreign bodies of the knee. KneeSurg Sports Traumatol Arthrosc. 2011 M ay;19(5):823-5.

Muschol M

7. , Drescher W, Petersen W, Hassenpflug J. M onarthritis of the pediatric knee joint: differential diagnosis after a thorn injury. Arthroscopy. 2004 Oct;20(8):865-8.

8. Kratz A, Greenberg D. Pantoeaagglomerans as a cause of septic arthritis after palm tree thorn injury: case report and literature review. Arch Dis Child. 2003;88:542-544.

9. Miller EB, Gilad A, Schattner A. Cactus thorn arthritis: case report and review of the literature. ClinRheumatol. 2000;19:490-491.

10. Olenginski TP, Bush DC, Harrington TM. Plant thorn synovitis: an uncommon cause on monoarthritis. Semin Arthritis Rheum. operating surgeon ${ }^{7}$. This led to the recommendation to remove all the macroscopically abnormal synovial lining. In most instances, this will involve complete synovectomy or recurrence of symptoms is possible $e^{1,7,10,16,19} . \mathrm{O}^{\prime}$ Connell $\mathrm{RL}$, Fageir M M, Addison A reported a case of synovitis of the knee due to trauma by a splinter of wood and also removal of the splinter by mini-arthrotomy ${ }^{20}$.Díaz-M artín AA et al; reported a case of arthroscopic removal of a projectile from the knee after a gunshot wound to the knee. ${ }^{21}$ Joris $\mathrm{F} . \mathrm{H}$. Duerinckx reported a case of subacute synovitis of knee after a rose thorn injury ${ }^{22}$. Muschol M, Drescher W, Petersen W, Hassenpflug J published on 'M onarthritis of the pediatric knee joint: differential diagnosis after a thorn injury. ' Wherein the misdiagnosis of a foreign body of knee was made as monoarthritis and arthroscopic removal of the foreign body was done after confirming the diagnosis?.

1991;21:40-46.

11. ZoltanJD. Cactusthorn synovitis.Arthroscopy. 1991;7:244-245.

12. Blake DR, Bacon PA, Scott CA, Potter AR. Monoarthritis from blackthorn injury: a novel means of diagnosis. Br M ed J (Clin Res Ed). 1981;282:361-362.

13. Freiberg AA, HerzenbergJE, Sangeorzan JA. Thorn synovitis of the knee joint with Nocardiapyarthrosis.ClinOrthopRelat Res. 1993;287:233-236.

14. Karshner RG, Hanafee W. Palm thorns as a cause of joint effusion in 17 children. Radiology. 1953;60:592-595.

15. Kelly JJ. Blackthorn inflammation. J Bone Joint Surg Br. 1966;48:474-477.

16. Sugarman M, Stobie DG, Quismorio FP, Terry R, Hanson V. Plant thorn synovitis. Arthritis Rheum. 1977;20:1125-1128.

17. Flatauer FE, Khan MA. Septic arthritis caused by Enterobacteragglomerans. Arch Intern Med. 1978;138:788.

18. Carandell M, Roig D, Benasco C. Plant thorn synovitis. J Rheumatol. 1980;7:567-569.

19. Doig SG, Cole WG. Plant thorn synovitis, resolution following total synovectomy. J Bone Joint Surg Br. 1990;72:514-515.

20. O'Connell RL, Fageir M M, Addison A. Be aware of wood in the knee. BMJ Case Rep. 2011 Dec 13;2011.

21. Díaz-Martín AA, Guerrero-M oyano N, Salinas-Sánchez P, GueradoParra E. [Arthroscopic removal of an intraarticular projectile from the knee]. ActaOrtop M ex. 2011 Jul-Aug;25(4):223-6. 